\title{
The clinical impact of epilepsy genetics
}

\begin{abstract}
An overemphasis on molecular genetic advances in epilepsy is in danger of missing the major contribution that clinical genetic studies make in predicting the likely benefit of molecular research efforts. Genetic epidemiology, twin, and family studies suggest that some individual epilepsy genes raise the risk for developing many different types of epilepsy but that specific combinations of these genes determine specific epilepsy phenotypes. Experimental data show how differences in drug response can result from inherited differences in drug sensitivity and there is emerging data on the genetic basis of harmful adverse drug reactions and teratogenesis. These developments predict a future in which epilepsy is ultimately classified on the basis of its oligogenic architecture, effective treatments are tailored to the appropriate patient and harmful adverse drug reactions avoided in those who are sensitive.
\end{abstract}

Epilepsy can be classified as idiopathic (where no aetiology other than a genetic predisposition is identified), symptomatic (epilepsy occurring as a result of identified brain lesion), and cryptogenic (where symptomatic epilepsy is suspected but an underlying brain lesion is not identified). With advances in genetics and neuroimaging the prevalence of cryptogenic epilepsy is falling.

The role of inheritance in epilepsy is traditionally categorised according to the mechanism of inheritance: (1) mendelian disorders in which epilepsy forms part of the phenotype; (2) idiopathic epilepsies with mendelian inheritance; (3) epilepsies with complex inheritance; (4) idiopathic epilepsies associated with cytogenetic abnormality.

There are over 200, individually rare, mendelian disorders in which epilepsy forms part of the phenotype. These include neurocutaneous disorders, neurodegenerative disorders, inherited malformations of cortical development, and an array of inherited metabolic disorders. Examples of recent interest are discussed elsewhere and although important, are not the focus of this editorial. ${ }^{1}$ Epilepsies with cytogenetic abnormality, as well as those demonstrating simple mendelian inheritance, are similarly rare and account for an estimated $1 \%$ of all epilepsy. Epilepsies in which the sole aetiology is a complex genetic predisposition are common and account for a minimum 50\% of all epilepsy.

\section{Defining the genetic component to epilepsy}

Familial aggregation of a disease, the principle measure of heritability, can result from shared genetic and environmental factors. Failure to show familial aggregation of a trait, or that familial aggregation results from shared genetic factors as opposed to shared environmental factors, may mean that the expense and effort of molecular studies is wasted. The genetic component of a disease refers to the extent of phenotypic variability that is under genetic control. The genetic component to epilepsy has been well established by the study of epilepsy in twins. ${ }^{2-4}$ In a rigorous, population based twin study, concordance for epilepsy in monozygotic (MZ) twin pairs was $62 \%$ compared with $18 \%$ in dizygotic (DZ) pairs. ${ }^{4}$ The high concordance for epilepsy amongst $M Z$ pairs and the relative magnitude of concordance of $\mathrm{MZ}$ pairs over $\mathrm{DZ}$ pairs indicates a strong genetic liability for epilepsy. Determining the number of genes involved in epilepsy from twin studies is rendered difficult by issues of sampling and genetic heterogeneity inherent to all twin studies. In principle, however, if concordance is $100 \%$ in $\mathrm{MZ}$ twin-pairs and $50 \%$ in $\mathrm{DZ}$ pairs, it may be concluded that the disease is strictly genetic and probably due to a single dominant gene. Similarly, an MZ concordance of $100 \%$ and a DZ concordance of $25 \%$ suggests either recessive inheritance or the interaction of two genes. For idiopathic generalised epilepsy (IGE), the estimated concordance among $M Z$ and DZ twin pairs of $76 \%$ and $33 \%$ respectively, ${ }^{4}$ suggests that IGE is a highly genetic condition and that only a few genes are involved in its aetiology. For IGE, the notable difference between DZ concordance (33\%) and offspring recurrence risk of IGE from a single affected parent $(4 \%),{ }^{5}$ suggests a role for recessive inheritance of sussceptibility in some people.

The role of genetic factors in the aetiology of postnatally acquired, remote symptomatic epilepsy seems to be minimal as the risk of epilepsy is not increased among relatives of probands with postnatal symptomatic epilepsy. ${ }^{6}$ By contrast, genetic factors are likely to be important in the aetiology of some cryptogenic epilepsies as there is an increased risk of epilepsy in relatives of probands with cryptogenic epilepsy ${ }^{6}$ and children with cryptogenic epilepsy and an affected first degree relative have a favourable prognosis consistent with an idiopathic aetiology. ${ }^{7}$ The likelihood that some cryptogenic partial epilepsies have a genetic, idiopathic aetiology, is born out in twin studies. ${ }^{8}$

\section{Defining the genetic component to epilepsy phenotypes}

Two important studies have examined the genetic basis to epilepsy phenotype. Berkovic tested the hypothesis that major epilepsy syndromes are distinct genetic entities determined by syndrome specific genes or combinations of genes. ${ }^{4}$ In this key study, $\mathrm{MZ}$ and $\mathrm{DZ}$ twin pairs concordant for epilepsy were found to be equally highly concordant for major epilepsy syndrome. Although the high concordance for epilepsy phenotype amongst $\mathrm{MZ}$ and $\mathrm{DZ}$ twin pairs concordant for epilepsy could have resulted from shared environmental factors, the biological implausibility of this argument strongly suggests that specific epilepsy genes determine both the presence of epilepsy and its specific clinical characteristics. This has clear practical importance in terms of the potential to accurately predict a person's epilepsy phenotype (and not just their risk of developing epilepsy) on the basis of their epilepsy genetic aetiology. Furthermore, twin studies suggest that the potential to predict epilepsy phenotype may extend to predicting epilepsy prognosis. Amongst twin pairs concordant for seizures and in whom one or other twin reported status 
Table 1 "Epilepsy genes"

\begin{tabular}{lrll}
\hline Gene & Locus & Epilepsy & Refs \\
\hline CHRNA4 & $20 \mathrm{q}$ & ADNFLE & 11 \\
CHRNB2 & $1 \mathrm{q}$ & ADNFLE & 12 \\
KCNQ2 & $20 \mathrm{q}$ & BFNC & 13,14 \\
KCNQ3 & $8 \mathrm{q}$ & BFNC & 15 \\
SCN1B & $19 \mathrm{q}$ & GEFS+ & 16 \\
SCN1A & $2 \mathrm{q}$ & GEFS+2 & 17
\end{tabular}

ADNFLE=autosomal dominant nocturnal frontal lobe epilepsy; $\mathrm{BFNC}=$ benign familial neonatal convulsions; GEFS+=generalised epilepsy with febrile seizures plus.

CHRNA4 is the gene for the $\alpha-4$ subnit of the ligand gated neuronal nicotinic acetylcholine receptor ( $\mathrm{nAChR}$ ). CHRNB2 is the gene for the $\beta-2$ subnit of nAChR. CHRNA4 and CHRNB2 gene products coassemble to form the most abundant $\mathrm{nAChR}$ subtype in mammalian brain. KCNQ2 and KCNQ3 are voltage gated neuronal potassium genes that form functional channels by heteromeric association with each other. SCN1B is the gene for the $\beta-1$ subunit and SCN1A is the gene for the $\alpha-1$ subunit of the voltage gated neuronal sodium channel.

epilepticus (SE), concordance for SE amongst $M Z$ twin pairs was greater than in $\mathrm{DZ}$ pairs, suggesting that genetic factors contribute risk for SE. ${ }^{9}$

The genetic basis for epilepsy phenotypes has also been considered using genetic epidemiology. ${ }^{10}$ Rate ratios for generalised and partial epilepsy were calculated in first degree relatives of 1498 adult probands with either partial or generalised epilepsy. Here, the authors found that the increased risk of epilepsy in first degree relatives was not necessarily restricted to the same type of epilepsy as in the proband. This finding provides evidence that some epilepsy genes have the potential to contribute to multiple epilepsy phenotypes, both partial and generalised. The (apparent) conflict with the twin data is reconciled by the hypothesis that specific combinations of epilepsy genes determine specific epilepsy phenotypes but that individually, some epilepsy genes have the potential to contribute to different types of epilepsy.

\section{Simple mendelian epilepsies are complex}

Although individually rare, the genetic analysis of "simple" mendelian epilepsies has been a key strategy in advancing our understanding of epilepsy. Six genes which, when mutated, may result in idiopathic epilepsy have been identified (table 1). Each of these genes encodes a subunit for an ion channel gated by voltage (KCNQ2, KCNQ3, $S C N 1 B, S C N 1 A)$ or a neurotransmitter (CHRNA4, $C H R N B 2)$. It is not known whether mutations or polymorphisms in these genes confer increased risk for more common epilepsies.

Despite disclosing idiopathic epilepsies as "ion channelopathies," identification of these genes has done little to explain the complex relation between genotype and epilepsy phenotype. It is already clear that specific epilepsy phenotypes can have more than one genetic cause. For example, mutations in both the $\alpha-4$ subunit gene (CHRNA4) and the $\beta-2$ subunit gene (CHRNB2) of the neuronal nicotinic acetylcholine receptor (nAChR) are associated with autosomal dominant nocturnal frontal lobe epilepsy (ADNFLE) ${ }^{11}{ }^{12}$ with a further locus mapped to chromosome $15 \mathrm{q} \cdot{ }^{18}$ Although the fact that mutations in CHRNB2 and CHRNA4 both result in ADNFLE can be readily explained in terms of the protein products of these two genes coassembling to form functional receptors, it is unlikely that a simple, structural relation between gene products will be a complete explanation for how mutations in different genes result in the same epilepsy phenotype.

In addition to specific epilepsy phenotypes having more than one genetic cause, it is also clear that specific genotypes can contribute to more than one epilepsy phenotype. For example, in the family epilepsy syndrome of generalised epilepsy with febrile seizures plus (GEFS+), affected persons with a specific SCN1A or SCN1B mutation manifest febrile seizures in association with a variety of afebrile seizure types including tonic-clonic, absence, myoclonic and atonic. ${ }^{19}$ Although partial seizures were not included in the original report of GEFS+, in the case of one GEFS + family, a single locus is seen to contribute to both partial and generalised seizure types; ${ }^{20}$ molecular genetic confirmation of a conclusion drawn previously from genetic epidemiology. ${ }^{10}$ How is this lack of correlation between genotype and phenotype explained?

In vitro expression studies of cloned ion channel genes have done little to provide an explanation. For example, the properties of two different CHRNA4 mutations from two phenotypically similar families were examined using reconstituted nAChRs in Xenopus oocytes. ${ }^{21}$ Both mutations result in major, but different changes in the physiological and pharmacological properties of the receptor, yet at the level of the epilepsy phenotype, have near identical effects. Again, how is this explained?

The explanation is concealed in a commonly held, but oversimplified view of "simple" Mendelian inheritance. Linkage analysis, which tracks a gene as it segregates through a pedigree, tracks only the "main effect" of a gene-that is, that which determines the average phenotypic effect taken above all other genes with which it may, or may not interact. Twenty years of linkage analysis in mendelian disorders has contributed to a widely held view that such disorders are caused by mutations in single genes. In reality, the situation is much more complex. ${ }^{22}{ }^{23}$ For example, the main effect of the SCN1B mutation is to raise the risk for the multiple seizure types that constitute the family epilepsy syndrome of GEFS+. Whether a person with this mutation develops seizures and what seizure type they develop is, however, determined by the interaction of the SCN1B mutation with other genetic and/or environmental factors. For this and other "simple" mendelian epilepsies, the phenotypes of patients are, in fact, complex traits. It is clear that accurate genotype-phenotype correlations will only come about when the complete genetic architecture of a person's epilepsy is revealed. This is the real challenge facing epilepsy genetics. An overemphasis on the causal relation of single genes to epilepsy is in danger of missing the major contribution other genetic factors make in determining a person's epilepsy phenotype.

\section{Epilepsy and pharmacogenetics}

Pharmacogenetics aims to predict therapeutic drug response and adverse drug reactions on the basis of inherited differences to drug sensitivity. ${ }^{24}$

Drug resistant epilepsy is a major problem and genetic mechanisms may contribute to refractoriness via pharmacodynamic or pharmacokinetic mechanisms. Alteration of the pharmacodynamic response to antiepileptic drugs (AEDs) might be influenced by genetic differences in receptor subunits and important evidence showing how the pharmacodynamic response to benzodiazepines can be genetically modified has been presented..$^{25}$ Pharmacokinetic mechanisms might also act to influence drug resistance in epilepsy. Multidrug resistance (MDR) is a term used to describe the phenomenon some tumours exhibit of simultaneous resistance to some structurally and functionally unrelated chemotherapeutic agents. ${ }^{27}$ The human MDR-1 gene encodes an integral membrane protein, $\mathrm{P}$-glycoprotein, the function of which is the energy dependent transport of molecules across membranes. Increased expression of P-glycoprotein in the brain of some patients with medically intractable epilepsy has been demonstrated suggesting that MDR proteins may play a part in determining the pharmacokinetic profile of AEDs within the blood-brain barrier. ${ }^{28}$ Functional polymorphisms in 
P-glycoprotein have been demonstrated ${ }^{29}$ and testing the hypothesis that such polymorphisms confer refractoriness to AEDs is an area of intense activity.

In addition to therapeutic drug response, certain adverse drug reactions may also have a genetic basis. Anticonvulsant hypersensitivity syndrome is a potentially fatal drug reaction with an estimated incidence of 1 in 10000 new drug exposures. ${ }^{30}$ Allergic sensitivity to toxic arene oxide intermediates is proposed as a possible mechanism ${ }^{31}$ and fatal anticonvulsant hypersensitivity syndrome occurring in a man with a functional epoxide hydrolase defect has been described..$^{32}$ Whereas this defect may have resulted from the anticonvulsant drug itself, sepsis, or treatment with corticosteroids, the potential for it to be genetically based is increased by the finding of familial aggregation of phenytoin hypersensitivity ${ }^{33}$ and concordance of carbamazepine hypersensitivity in MZ twins. ${ }^{34}$ However, a single mutation within the coding region of the microsomal epoxide hydrolase gene is unlikely to be the sole determinant of this genetic predisposition. ${ }^{35}$

Finally, the teratogenic effects of anticonvulsant drugs are well known and the potential for pharmacogenetics to stratify offspring risk on the basis of genetic susceptibility is long recognised. ${ }^{36}$ More recently, the identification of inbred strains of mice which are more or less sensitive to the teratogenic effects of sodium valproate has lent support to the notion that offspring liability to valproate induced teratogenicity has a genetic basis and offers the potential to identify high risk couplings. ${ }^{37}$ The magnitude of risk conferred by genetic factors, however, remains to be determined. If such genes confer low relative risk for a person (despite a potentially high population attributable risk) then intervention based on the identification of risk conferring alleles may not be warranted.

\section{Conclusion}

A hereditary component to epilepsy has been suspected since the time of Hippocrates, but only in the past few years has the genetic basis for this liability begun to unravel. Spectacular advances have suggested mechanisms of epileptogenesis hitherto unconsidered. Experimental data demonstrate how differences in drug response can result from inherited differences in drug sensitivity and there are emerging data on the genetic basis of harmful adverse drug reactions and teratogenesis. These developments predict a future in which epilepsy is ultimately classified on the basis of its oligogenic architecture and pharmacogenetics tailors the appropriate drug to the appropriate patient, with attendant benefits in both clinical practice and cost.

This work has been supported by an educational grant from Jansen-Cilag. We are grateful to Drs MC Walker and NW Wood for the critical reading of this manuscript.

M R JOHNSON

Division of Neuroscience and Psychological Medicine, Charing Cross Hospital, Fulham Palace Road, London W6 8RF, UK

M R JOHNSON

J W A S SANDER

University College London Institute of Neurology, The National Hospital for Neurology and Neurosurgery and Institute of Neurology, Queen Square, London WC1N 3BG, UK

J W A S SANDER National Society of Epilepsy, Chalfont Centre for Epilepsy, Chalfont St Peter, Bucks SL9 ORF, UK

Correspondence to: Dr M R Johnson

M.johnson@ion.ucl.ac.uk

1 Berkovic SF, Scheffer IE. Genetics of the epilepsies. Curr Opin Neurol 1999;12:177-82.
2 Lennox WG. The heredity of epilepsy as told by relatives and twins. $7 A M A$ 1951;146:529-36.

3 Anderson VA, Wilcox KJ, Leppik LE, et al. Twin studies in epilepsy. In: Beck-Managetta G, Anderson VE, Doose H, eds. Genetics of the epilepsies. Heidelberg: Springer-Verlag, 1989:145-55.

4 Berkovic SF, Howell RA, Hay DA, et al. Epilepsy in twins: genetics of the major epilepsy syndromes. Ann Neurol 1998;43:435-45.

5 Anderson VA, Hauser WA. The genetics of epilepsy. In: Bearn A G, Childs B, Motulsky A, eds. Progress in medical genetics VI. Philadelphia: Saunders, 1985.

6 Ottman R, Annegers JF, Risch $\mathrm{N}$, et al. Relations of genetic and environmental factors in the etiology of epilepsy. Ann Neurol 1996;39:4429.

7 MacIntosh DS, Camfield PR, Camfield CS. Children with familial crypogenic epilepsy have a favourable seizure prognosis. $f$ Child Neurol 1998;13:372-6.

8 Berkovic SF, McIntosh A, Howell RA, et al. Familial temporal lobe epilepsy: a common disorder identified in twins. Ann Neurol 1996;40:227-35.

9 Corey LA, Pellock JM, Boggs JG, et al. Evidence for a genetic predisposition for status epilepticus. Neurology 1998;50:558-60.

10 Ottman R, Lee JH, Hauser A, et al. Are generalised and localisation-related epilepsies genetically distinct? Arch Neurol 1998;55:339-44.

11 Steinlein OK, Mulley JC, Propping P, et al. A missense mutation in the neuronal nicotinic acetylcholine receptor alpha 4 subunit is associated with autosomal dominant nocturnal frontal lobe epilepsy. Nat Genet 1995;11: 201-4.

12 De Fusco M, Becchetti A, Patrignani A, et al. The nicotinic receptor B2 subunit is mutant in nocturnal fronal lobe epilepsy. Nat Genet 2000;26:275-6.

13 Singh NA, Charlier C, Stauffer D, et al. A novel potassium channel gene, KCNQ2, is mutated in an inherited epilepsy of newborns. Nat Genet 1998; 18:25-9.

14 Biervert C, Schroeder BC, Kubisch C, et al. A potassium channel mutation in neonatal human epilepsy. Science 1998;279:403-6.

15 Charlier C, Singh NA, Ryan SG, et al. A pore mutation in a novel KQT-like potassium channel gene in an idiopathic epilepsy family. Nat Genet 1998;18:53-5.

16 Wallace RH, Wang DW, Singh R, et al. Febrile seizures and generalised epilepsy associated with a mutation in the $\mathrm{Na}+$ channel beta 1 subunit gene SCN1B. Nat Genet 1998;19:366-70.

17 Escayg A, MacDonal BT, Meisler MH, et al. Mutations of SCN1A, encoding a neuronal sodium channel, in two families with GEFS+2. Nat Genet 2000;24:343-5.

18 Philips HA, Scheffer IE, Chinvarun Y, et al. Autosomal dominant nocturnal frontal lobe epilepsy: genetic heterogeneity and evidence for a second locus at 15q24. Am f Hum Genet 1998;63:1108-16.

19 Singh R, Scheffer IE, Crossland K, et al. Generalised epilepsy with febrile seizures plus: a common childhood onset genetic epilepsy syndrome. Ann Neurol 1999;45:75-81.

20 Baulac S, Gourfinkel-An I, Picard F, et al. A second locus for familial generalised epilepsy syndromes with febrile seizures plus maps to chromosome 2q21-q33. Am ₹ Hum Genet 1999;65:1078-85.

21 Bertrand S, Weiland S, Berkovic SF, et al. Properties of neuronal nicotinic acetylcholine receptor mutants from humans suffering from autosomal dominant nocturnal frontal lobe epilepsy. Br f Pharmacol 1998;125:75160

22 Dipple KM, McCabe ERB. Phenotypes of patients with simple mendelian disorders are complex traits: thresholds, modifiers and systems dynamics. Am F Hum Genet 2000;66:1729-35.

23 Weatherall DJ. Single gene disorders or complex traits: lessons from the thalassaemias and other monogenic diseases. BMF 2000;321:1117-20.

24 Marshall A. Laying the foundations for personalized medicines. Nature Biotechnology 1998:16:6-8.

25 Rudolph U, Crestani F, Benke D, et al. Benzodiazepine actions mediated by specific $\gamma$-aminobutyric acid (A) receptor subtypes. Nature 1999;401:796800.

26 McKernan RM, Rosahl TW, Reynolds DS, et al. Sedative but not anxiolytic properties of benzodiazepines are mediated by the GABAA receptor $\alpha 1$ subtype. Nat Neurosci 2000;3:587-92.

27 Krishna R, Mayer LD. Multidrug resistance (MDR) in cancer. Mechanisms, reversal using modulators of MDR and the role of MDR modulators in influencing the pharmacokinetics of anticancer drugs. Eur F Pharm Sci 2000;11:265-83

28 Tishler DM, Weinberg KI, Hinton DR, et al. MDR1 gene expression in brain of patients with medically refractory epilepsy. Epilepsia 1995;36:1-6.

29 Hoffmeyer S, Burk O, von Richter O, et al. Functional polymorphisms of the human multidrug-resistance gene: multiple sequence variations and correlation of one allele with P-glycoprotein expression and activity in vivo. Proc Nat Acad Sci 2000;97:3473-8.

30 Vittorio CC, Muglia JJ. Anticonvulsant hypersensitivity syndrome. Arch Intern Med 1995;115:2285-90.

31 Shear NH, Spielberg SP. Anticonvulsant hypersensitivity syndrome: in vitro assessment of risk. $\mathcal{F}$ Clin Invest 1988;82:1826-32.

32 Yoo JH, Kang DS, Chun WH, et al. Anticonvulsant hypersensitivity syndrome with an epoxide hydrolase defect. Br f Dermatol 1999;140:181 3 .

33 Gennis MA, Vemuri R, Burns EA, et al. Familial occurrence of hypersensitivity to phenytoin. Am F Med 1991;91:631-4.

34 Edwards SG, Hubbard V, Aylett S, et al. Concordance of primary generalised epilepsy and carbamazepine hypersensitivity in monozygotic twins. Postgrad Med f 1999;75:680-1.

35 Green VJ, Pirmohammed M, Kitteringham NR, et al. Genetic analysis of microsomal epoxide hydrolase in patients with carbamazepine hypersensitivity. Biochem Pharm 1995;50:1353-9.

36 Lindhout D. Pharmacogenetics and drug interactions: role in antiepilepticdrug induced teratogenesis. Neurology 1992;42:(suppl 5):43-7.

37 Faiella A, Wernig M, Consalez GG, et al. A mouse model for valproate teratogenicity: parental effects, homeotic transformations and altered HOX expression. Hum Mol Gen 2000;9:227-36. 